\title{
A REPRESENTATION OF m-ALGEBRAIC LATTICES
}

\author{
G. GRÄTZER, P. M. JOHNSON, AND E. T. SCHMIDT
}

\begin{abstract}
In this paper, we give a short proof of the following result of G. Grätzer and E. T. Schmidt: every m-algebraic lattice can be represented as the lattice of $\mathfrak{m}$-complete congruence relations of some $\mathfrak{m}$-complete modular lattice.
\end{abstract}

\section{INTRODUCTION}

In 1983 TR. Wille raised the following question (seeTe.g. TK. Reuter and R. Wille [7]): Is every complete lattice $L$ isomorphic to the lattice of complete congruence relations of a suitable complete lattice $K$ ? S.-K. Teo [8] solved this problem for a finite lattice $L$. An affirmative solution to the question was provided in G. Grätzer [5]T where the background of this field was also discussed. In a series of papersTvarious authors obtained sharper results (see [6] for a detailed accounting) Tculminating in the following result of G. Grätzer and E. T. Schmidt [6]:

Theorem . Let $\mathfrak{m}$ be a regular cardinal $>\aleph_{0}$. Every $\mathfrak{m}$-algebraic lattice $L$ is isomorphic to the lattice of $\mathfrak{m}$-complete congruence relations of a suitable $\mathfrak{m}$-complete modular lattice $K$.

In this paper $\Gamma$ we shall present a new proof of this result. Given $L \Gamma$ a suitable lattice $K$ is constructed in a short and direct way which allows easy study of its $\mathfrak{m}$-complete congruences. It can be shown-although we will not do this - that the construction of [6] yields the same lattice $K$.

\section{Notation}

A cardinal $\mathfrak{m}$ is regular if whenever $J$ is a set with $|J|<\mathfrak{m}$ and $\left(I_{j} \mid j \in J\right)$ is a family of sets satisfying $\left|I_{j}\right|<\mathfrak{m} \Gamma$ for all $j \in J \Gamma$ then $\left|\bigcup\left(I_{j} \mid j \in J\right)\right|<\mathfrak{m}$. For instance $\Gamma$ all cardinals of the form $\aleph_{\alpha+1}$ are regular. In this paper $\Gamma \mathrm{m}$ stands for a fixed uncountable regular cardinal. A set of cardinality less than $\mathfrak{m}$ is said to be small. We refer the reader to [3] and [4] for standard lattice-theoretic notation and for proofs of elementary facts about algebraic lattices; natural extensions to $\mathfrak{m}$-algebraic lattices $\Gamma$ which first appeared in [2] Tare restated below.

Some definitions and remarks have analogues for $\mathfrak{m}=\aleph_{0}$ Tbut this is not the case for our later results. In the Theorem itselfTthe requirement that $\mathfrak{m}>\aleph_{0}$ is essential

Date: Final version: November 8, 1991.

1991 Mathematics Subject Classification. Primary 06B10; Secondary 06C05.

Key words and phrases. Complete lattice, modular lattice, complete congruence, congruence lattice, $\mathfrak{m}$-complete lattice, $\mathfrak{m}$-algebraic lattice, $\mathfrak{m}$-complete congruence.

The research of the first author was supported by the NSERC of Canada.

The research of the third author was supported by the Hungarian National Foundation for Scientific Research, under Grant No. 1903. 
because the lattice Con $K$ of $\aleph_{0}$-complete congruences of a lattice $K$ is distributive $\Gamma$ and so a non-distributive algebraic lattice $L$ cannot be represented in that form.

A lattice $M$ is $\mathfrak{m}$-complete if $\bigvee X$ and $\bigwedge X$ exist in $M$ for every small nonempty subset $X$ of $M$. A congruence relation $\Theta$ of an $\mathfrak{m}$-complete lattice $M$ is an $\mathfrak{m}$-complete congruence relation if the Substitution Property holds for fewer than $\mathfrak{m}$ elements; that is $\Gamma$ if $x_{j} \equiv y_{j}(\Theta)$ for all $j$ in some nonempty small set $J \Gamma$ then

$$
\bigvee\left(x_{j} \mid j \in J\right) \equiv \bigvee\left(y_{j} \mid j \in J\right)
$$

and

$$
\bigwedge\left(x_{j} \mid j \in J\right) \equiv \bigwedge\left(y_{j} \mid j \in J\right) \quad(\Theta) .
$$

An element $c$ of a lattice $M$ is $\mathfrak{m}$-compact if $c \leq \bigvee X$ implies that $c \leq \bigvee X_{1}$ for some small subset $X_{1}$ of $X$. A lattice $M$ is $\mathfrak{m}$-algebraic if it is complete (not just $\mathfrak{m}$-complete) and every element is a join of $\mathfrak{m}$-compact elements.

The lattice $\operatorname{Con}_{\mathfrak{m}} M$ of all $\mathfrak{m}$-complete congruence relations of an $\mathfrak{m}$-complete lattice $M$ is an $\mathfrak{m}$-algebraic lattice (see [2]); its lattice operations are denoted by $\wedge \Gamma$ $\vee_{\mathfrak{m}}$ Tand the non-binary variants by $\Lambda \Gamma \bigvee_{\mathfrak{m}}$. Note that $\wedge$ and $\Lambda$ are set intersection.

We always let $L$ denote the $\mathfrak{m}$-algebraic lattice in the Theorem; $K$ is the lattice we construct to prove the Theorem. If $L$ has only one element $\Gamma$ we take $K=L$. We shall henceforth assume that $|L| \geq 2$.

Let $C$ denote the set of nonzero $\mathfrak{m}$-compact elements of $L$. Since $|L| \geq 2 \Gamma$ it follows that $C \neq \varnothing$. The set $C$ inherits a partial order from $L$ and it is closed under small nonempty joins in $L$. Since $C$ need not have a least element $\Gamma$ it is not $\Gamma$ in general $\Gamma$ an $\mathfrak{m}$-complete join-semilattice.

An $\mathfrak{m}$-complete ideal of $C$ is a nonempty subset $I$ of $C$ with the property that $\Gamma$ for each small nonempty subset $X$ of $C \Gamma \bigvee X \in I$ iff $X \subseteq I$. In particularTif $c \in I$ and $c^{\prime} \in C$ with $c^{\prime}<c \Gamma$ then $c^{\prime} \in I$ Tas can be seen by using $X=\left\{c, c^{\prime}\right\}$. Let $\operatorname{Id}_{\mathfrak{m}} C$ denote the set $\Gamma$ ordered by inclusion $\Gamma$ of all $\mathfrak{m}$-complete ideals of $C \Gamma$ together with

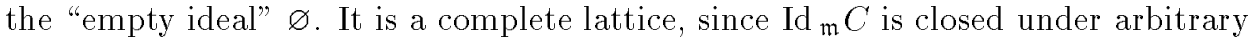
intersections.

The importance of $C$ in the proof of the Theorem stems in part from the following easy result (see [2]).

Lemma 1. The map $x \mapsto\{c \in C \mid c \leq x\}$ is an isomorphism between $L$ and $\operatorname{Id}_{\mathfrak{m}} C$.

When discussing posets (partially ordered sets) $\Gamma$ we usually let $\leq$ denote the (partial) order $\Gamma$ with $<$ standing for the corresponding strict order. An ordinal is a poset with the structure of a well-ordered chain. The sum of the posets $A_{1}$ and $A_{2}$ will be denoted by $A_{1}+A_{2}$; we place $A_{2}$ on top of $A_{1}$. Define $2 A_{1}=A_{1}+A_{1}$. The lexicographic product of the posets $A_{1}$ and $A_{2}$ is the set $A_{1} \times A_{2}$ Tordered by

$$
\left\langle a_{1}, a_{2}\right\rangle \leq\left\langle a_{1}^{\prime}, a_{2}^{\prime}\right\rangle \text { iff } a_{1}<a_{1}^{\prime} \text { or } a_{1}=a_{1}^{\prime} \text { and } a_{2} \leq a_{2}^{\prime} .
$$

For any poset $A \Gamma$ let $A^{*}$ denote the lexicographic product of the chain $\omega=$ $\{0,1,2, \ldots\}$ with $A$; it has no largest element. If $A$ is a well-ordered chain $\Gamma$ then so is $A^{*}$.

Let $\left\{A_{\gamma} \mid \gamma<\chi\right\}$ be the set of all small nonempty subsets of $C$ Tindexed by the ordinal $\chi$. Henceforth $\Gamma \chi$ always stands for this ordinal; $\chi$ is not $0 \Gamma$ since $C$ is nonempty. We assume that each $A_{\gamma} \Gamma \gamma<\chi$ Thas been well-ordered. 
In preparation for in the construction of the lattice $K$ Twe define the chains $B_{\gamma} \Gamma$ $\gamma<2 \chi$ Tas follows:

For $\gamma<\chi$ Tlet $B_{\gamma}=A_{\gamma}^{*}$.

For $\chi \leq \gamma<2 \chi$ Tlet $B_{\gamma}=\omega \times\{\gamma\}$ be a chain isomorphic to $\omega$ Twith the elements

$$
\langle 0, \gamma\rangle\langle\langle 1, \gamma\rangle<\langle 2, \gamma\rangle<\ldots
$$

Let $B$ be the disjoint union $\bigcup^{\prime}\left(B_{\gamma} \mid \gamma<2 \chi\right)$. From the strict linear orders on the $B_{\gamma} \Gamma \gamma<2 \chi$ Twe define a strict partial order on $B \Gamma$ denoted by $\ll$ (to avoid confusion with other partial orders). For $b_{1} \Gamma b_{2} \in B \Gamma$ say $b_{1} \in B_{\gamma}$ and $b_{2} \in B_{\gamma^{\prime}} \Gamma$

$$
b_{1} \ll b_{2} \text { iff } \gamma=\gamma^{\prime} \text { and } b_{1}<b_{2} \text { in } B_{\gamma} \text {. }
$$

This gives $B$ the structure of a collection of mutually incomparable small wellordered chainsTeach having no largest element.

There is a natural pairing of these chains Tand of the ordinals less than $2 \chi$ Tgiven by the operation :

For $\gamma<\chi$ Tlet $\tilde{\gamma}=\chi+\gamma$

For $\chi \leq \gamma<2 \chi$ Tlet $\tilde{\gamma}$ be the unique ordinal satisfying $\chi+\tilde{\gamma}=\gamma$.

So $\Gamma$ for any $\gamma<2 \chi$ Tthe operation interchanges $\gamma$ and $\tilde{\gamma}$.

The elements of $C$ are called colors Tand a coloring of a set $X$ is a function from $X$ onto $C$. A coloring of $X$ partitions $X$ into color classes $X^{c}=\{b \in B \mid \varphi(b)=c\} \Gamma$ where $c \in C$. Coloring is a technique introduced by $\mathrm{S}$. $\mathrm{K}$. Teo [8].

The construction of $K$ uses the following coloring $\varphi: B \rightarrow C$. Let $b \in B$ say $b \in B_{\gamma} \Gamma$ where $\gamma<2 \chi$. Then define

$$
\varphi(b)= \begin{cases}a, & \text { if } \gamma<\chi \text { and } b=\langle i, a\rangle \\ \bigvee A_{\tilde{\gamma}}, & \text { if } \chi \leq \gamma<2 \chi\end{cases}
$$

Recall that any $A_{\gamma^{\prime}} \Gamma \gamma^{\prime}<\chi$ Thas a join in $C$.

Henceforth $\Gamma$ the coloring of $B$ will always be this coloring; the color classes $B^{c} \Gamma$ $c \in C$ Tare the color classes under this coloring. Note that $B^{c} \neq \varnothing \Gamma$ for all $c \in C$.

\section{The lattice $K$}

Let $V$ be a vector space. The set of all subspaces of $V$ Tordered by inclusionTis denoted by $\mathrm{PG}(V)$. It is a complete lattice in which meet is set intersection and the join of the two subspaces $s$ and $t$ is $s+t$. A lattice isomorphic to a $\mathrm{PG}(V)$ is a projective geometry. (There are other projective geometries which we need not consider here.) Note that any interval sublattice $[s, t]$ of a projective geometry is again a projective geometry. We also use the lattice-theoretic concept of projectivity $\Gamma$ and the following easily established fact: In a projective geometry, any two prime intervals are projective to each other.

For a set $X$ of vectors of $V$ Tlet $[X]$ denote the subspace of $V$ spanned by $X$. If $S$ is a set of of subspaces of $V$ such that $[\bigvee(S \backslash\{s\})] \cap s=\{0\}$ Tfor all $s \in S \Gamma$ then $[\bigcup S]$ will be denoted by $\bigoplus S$.

We shall construct the lattice $K$ as a sublattice of a projective geometry $\mathrm{PG}(W) \Gamma$ where $W$ is any vector space with basis $B$ (the set $B$ was defined in Section 2). The choice of the underlying field (or division ring) is immaterial; one may use the two-element fieldTas in [6]. 
Any $v \in W$ can be represented in the form $v=\sum_{b} \lambda_{b} b$ Twhere $b \in B$ and $\lambda_{b}$ is an element of the underlying field. The set

$$
\operatorname{supp} v=\left\{b \in B \mid \lambda_{b} \neq 0\right\}
$$

is finite; it is called the support of $v$.

For any linear combination $v=\sum\left(\lambda_{i} v_{i} \mid 1 \leq i \leq n\right)$ of vectors in $W \Gamma$ we have:

$$
\operatorname{supp} v \subseteq \bigcup\left(\operatorname{supp} v_{i} \mid 1 \leq i \leq n\right) \text {. }
$$

As always $\Gamma C$ is the set of nonzero m-compact elements of $L$. The variables $b \Gamma c \Gamma$ and $v$ range over $B \Gamma C$ Tand $W$ Trespectively $\Gamma$ while $s \Gamma t \Gamma$ and so on $\Gamma$ denote subspaces of $W$.

The decomposition of $B$ into color classes induces the decompositions

$$
W=\bigoplus\left(\left[B^{c}\right] \mid c \in C\right)
$$

and

$$
v=\sum\left(v^{c} \mid c \in C\right),
$$

where $v^{c} \in\left[B^{c}\right]$ and all but finitely many of the vectors $v^{c}$ are 0 . For any subspace $s$ of $W \Gamma$ we also write $s^{c}=s \cap\left[B^{c}\right]$. Note that $s \supseteq \bigoplus\left(s^{c} \mid c \in C\right) \Gamma$ where the containment is $\Gamma$ in general $\Gamma$ strict.

Let $K$ be the set of all subspaces $s$ of $W$ satisfying the following conditions:

(K1) $\operatorname{dim} s<\mathfrak{m}$.

(K2) $s=\bigoplus\left(s^{c} \mid c \in C\right)$.

(K3) If $v \in s \Gamma b_{1} \in \operatorname{supp} v \Gamma$ and $b_{2} \in B$ with $b_{2} \ll b_{1} \Gamma$ then $b_{2} \in s$.

(K4) For $\gamma<2 \chi \Gamma B_{\gamma} \subseteq s$ iff $B_{\tilde{\gamma}} \subseteq s$.

The lattice $K$ has a zero element $0_{K}$ Tnamely the subspace $\{0\}$ of $W$.

If the elements $s_{i} \Gamma i \in I$ Tof PG(W) satisfy (K2) (in particularTif they lie in $K$ ) $\Gamma$ then

$$
\left(\bigvee\left(s_{i} \mid i \in I\right)\right)^{c}=\bigvee\left(s_{i}^{c} \mid i \in I\right) \text { and }\left(\bigwedge\left(s_{i} \mid i \in I\right)\right)^{c}=\bigwedge\left(s_{i}^{c} \mid i \in I\right),
$$

for all $c \in C$.

Lemma 2. $K$ is a sublattice of $\mathrm{PG}(W)$; it is an $\mathfrak{m}$-complete lattice.

Proof. It is obvious that $K$ is closed under arbitrary nonempty meets formed in PG(W). Hence $\Gamma$ to prove the lemmaTwe must verify two statements: $K$ is closed under finite joins formed in $\mathrm{PG}(W)$; and small joins exist in $K$.

To verify the first statement $\Gamma$ let $s \Gamma t \in K$. We show that $s \vee t$ satisfies (K1)-(K4). (K1) is obvious. By (4) $\Gamma(\mathrm{K} 2)$ holds for $s \vee t$. Let $v \in s \vee t \Gamma$ say $v=v_{1}+v_{2}$ Twhere $v_{1} \in s$ and $v_{2} \in t$. Since $s$ and $t$ satisfy (K3) $\Gamma$ so does $s \vee t \Gamma$ by (3).

FinallyTsuppose that $B_{\gamma} \subseteq s \vee t$ for some $\gamma<2 \chi$; we wish to show that $B_{\tilde{\gamma}} \subseteq s \vee t$. We claim that $B_{\gamma} \subseteq s$ or $B_{\gamma} \subseteq t$. Assume to the contrary that $b_{1} \Gamma b_{2} \in B_{\gamma}$ and $b_{1} \notin s \Gamma b_{2} \notin t$. Then we can choose $b \in B_{\gamma}$ with $b_{1} \ll b \Gamma b_{2} \ll b$. By (3) $\Gamma b$ is in the support of an element of $s$ or of $t \Gamma$ contradicting (K3) for $s$ or $t$. So $B_{\gamma} \subseteq s$ or $B_{\gamma} \subseteq t$. Since $s$ and $t$ satisfy (K4) $\Gamma$ we conclude that $B_{\tilde{\gamma}} \subseteq s$ or $B_{\tilde{\gamma}} \subseteq t$ Twhich implies that $B_{\tilde{\gamma}} \subseteq s \vee t$. This proves (K4) for $s \vee t$. Therefore $s \vee t \in K$. 
To verify the second statement $\Gamma$ let $S$ be a small subset of $K$. In $\mathrm{PG}(W) \Gamma$ let $u^{\prime}=\bigvee S$. Define

$$
\Gamma_{u^{\prime}}=\left\{\gamma \mid \gamma<2 \chi, B_{\gamma} \nsubseteq u^{\prime}, B_{\tilde{\gamma}} \subseteq u^{\prime}\right\} .
$$

Since the $B_{\gamma}$ are pairwise disjoint and $\operatorname{dim} u^{\prime}<\mathfrak{m} \Gamma$ it follows that $\Gamma_{u^{\prime}}$ is small. Therefore $\Gamma$ the set

$$
u=\left[u^{\prime} \cup \bigcup\left(B_{\gamma} \mid \gamma \in \Gamma_{u^{\prime}}\right)\right]
$$

satisfies (K1) as well as (K4). Using (3) and (4) $\Gamma$ it is easy to see that $u$ satisfies (K2).

To verify (K3) $\Gamma$ let $v \in u \Gamma b_{1} \in \operatorname{supp} v \Gamma$ and $b_{2} \ll b_{1}$ in $B$. We have at least one of: (i) $b_{1} \in \operatorname{supp} v^{\prime}$ Tfor some $v^{\prime} \in u^{\prime}$ Tor (ii) $b_{1} \in B_{\gamma} \Gamma$ for some $\gamma \in \Gamma_{u^{\prime}}$.

If (i) holds $\Gamma$ then we apply (3) to obtain $b_{1} \in \operatorname{supp} v^{\prime \prime} \Gamma$ where $v^{\prime \prime} \in s \Gamma$ for some $s \in S$. Then (K2) and (K3) imply that $b_{2} \in s$ Tand therefore $b_{2} \in u$.

If (ii) holds and $b_{1}$ lies in $B_{\gamma} \Gamma$ then so does $b_{2} \Gamma$ by the definition of the relation $\ll$ in (1). Hence $b_{2} \in u$.

This completes the verification of (K1)-(K4) for $u$. Therefore $u \in K$. Moreover $\Gamma$ $u$ is clearly the join in $K$ of $S$. Thus $K$ is an m-complete lattice.

Next Twe show that certain intervals in $K$ are projective geometries.

Lemma 3. Let $s \in K$. For some color $c$, let $X$ be a small subset of $B^{c}$ satisfying the condition:

$$
\text { If } b_{1} \in X \text { and } b_{2} \ll b_{1} \text {, then } b_{2} \in s \text {. }
$$

Then $t \in K$ for any subspace $t$ of $W$ such that $s \subseteq t \subseteq s \vee[X]$.

Proof. By modularity $t=s \vee(t \cap[X])$. Of course $t$ satisfies (K1). As $s$ satisfies (K2) $\Gamma$ it has a basis consisting of elements of $\bigcup\left(\left[B^{c}\right] \mid c \in C\right)$. Now $t$ has the same propertyГsince $t \cap[X] \subseteq\left[B^{c}\right] \Gamma$ so $t$ satisfies (K2). Let $b_{1}$ be an arbitrary element of $B$ lying in the support of a vector of $t$. We must verify (K3) with $t$ in place of $s$ and with the $b_{1}$ just defined. Note that $b_{1}$ is either an element of $X$ or else it lies in the support of a vector of $s$. In the first case $\Gamma(\mathrm{K} 3)$ holds by (7); in the second case $\Gamma$ it holds because $s$ satisfies (K3). Since $B_{\gamma}$ has no greatest elementTit follows

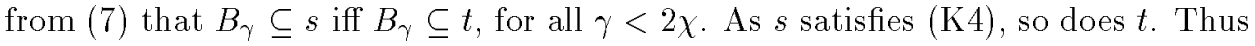
$t \in K$.

Lemma 4. Every prime interval $[s, t]$ of $K$ is prime in $\mathrm{PG}(W)$, and there is a unique color $c$ with $s^{c} \neq t^{c}$.

Proof. Let $[s, t]$ be a prime interval of $K$. If $s \cap B \neq t \cap B \Gamma$ then choose $v$ as an element in $\langle B ; \ll\rangle$ which is minimal subject to $v \in t \backslash s$. If $s \cap B=t \cap B$ Then we can $\Gamma y$ by $(\mathrm{K} 2) \Gamma$ choose $v \in t \backslash s$ with the property that $v \in\left[B^{c}\right]$ for some color $c$. Note that $t$ satisfies (K3). Therefore $\Gamma X=\operatorname{supp} v$ is a nonempty finite set satisfying (7). Define $t_{1}=[s \cup\{v\}]$. By Lemma $3 \Gamma t_{1} \in K$ and hence $t_{1}=t$. Evidently $\Gamma[s, t]$ is a prime interval of $\mathrm{PG}(W)$ and $s^{c} \neq t^{c}$. As $s$ satisfies (K2) and $v \in\left[B^{c}\right]$ Twe also have $s^{c^{\prime}}=t^{c^{\prime}}$ whenever $c^{\prime} \neq c$.

The previous lemma allows us to color the prime intervals of $K$ in a natural way: the color of $[s, t]$ is the unique color $c$ with $s^{c} \neq t^{c}$. Given $b \in B$ Twe can define a prime interval $\left[s_{b}, t_{b}\right]$ of $K$ whose color is $\varphi(b)$ Tas follows:

$$
s_{b}=\left[\left\{b_{1} \in B \mid b_{1} \ll b\right\}\right], t_{b}=\left[s_{b} \cup\{b\}\right] .
$$


Such prime intervals play a crucial role in the proof $\mathrm{starting}$ with the next two lemmas.

Lemma 5. Every prime interval of $K$ is projective to one of the form $\left[s_{b}, t_{b}\right]$, where $b \in B$.

Proof. In $K$ Tlet $[s, t]$ be a prime interval of color $c$. Let $X=\operatorname{supp} v$ Twhere $v \in t \backslash s$ is chosen as in the proof of Lemma 4. Then $X$ satisfies condition (7) Tand $X \nsubseteq s$. Choose $b \in X \backslash s$ and set $t^{\prime}=[s \cup\{b\}]$. Note that $s_{b} \leq s$ and $t_{b} \leq t^{\prime} \Gamma$ by $(7)$ and the definitions of $s_{b} \Gamma t_{b} \Gamma$ and $t^{\prime}$.

Lemma 3 implies that all subspaces of $W$ between $s$ and $[s \cup X]$ lie in $K$; therefore the prime intervals $[s, t]$ and $\left[s, t^{\prime}\right]$ are projective in $K$. The latter interval $\Gamma a n d$ hence the former $\Gamma$ is projective to $\left[s_{b}, t_{b}\right]$ because $s \vee t_{b}=t^{\prime}$ and $s \wedge t_{b}=s_{b}$.

Lemma 6. Two prime intervals of $K$ are projective iff they have the same color.

Proof. If $s \Gamma t$ Tand $u$ are elements of $K$ and $s^{c}=t^{c} \Gamma$ for some color $c$ Tthen $(s \vee u)^{c}=$ $(t \vee u)^{c}$ and $(s \wedge u)^{c}=(t \wedge u)^{c} \Gamma$ by (4). In particularTprojective prime intervals of $K$ have the same color.

To establish the converse $\Gamma$ it suffices $\Gamma$ by Lemma 5 Tto prove that if $b$ and $b^{\prime} \in B$ are of the same color $c \Gamma$ then the prime intervals $\mathfrak{p}=\left[s_{b}, t_{b}\right]$ and $\mathfrak{p}^{\prime}=\left[s_{b^{\prime}}, t_{b^{\prime}}\right]$ are projective. Let $b \in B_{\gamma} \Gamma b^{\prime} \in B_{\gamma^{\prime}} \Gamma$ where $\gamma \Gamma \gamma^{\prime}<2 \chi$. We can reduce this proof to the case $\gamma \neq \gamma^{\prime}$ by using the transitivity of projectivity and by choosing some $\mathfrak{p}^{\prime \prime}=\left[s_{b^{\prime \prime}}, t_{b^{\prime \prime}}\right]$ of color $c \Gamma$ where $b^{\prime \prime} \in B_{\gamma^{\prime \prime}}$ and $\gamma^{\prime \prime} \neq \gamma$. Such a $\mathfrak{p}^{\prime \prime}$ always exists.

Define $s=s_{b} \vee s_{b^{\prime}} \Gamma t=s \vee t_{b}$ and $t^{\prime}=s \vee t_{b^{\prime}}$. Note that $t=[s \cup\{b\}] \Gamma$ and $t^{\prime}=\left[s \cup\left\{b^{\prime}\right\}\right]$. Since $\gamma \neq \gamma^{\prime} \Gamma \mathfrak{p}$ and $\mathfrak{p}^{\prime}$ are projective in $K$ to the prime intervals $[s, t]$ and $\left[s, t^{\prime}\right]$ Trespectively. By applying Lemma 3 with $s=s_{b} \vee s_{b^{\prime}}$ and $X=\left\{b, b^{\prime}\right\} \Gamma$ we conclude that $\left[s, t \vee t^{\prime}\right]$ is a projective geometry that lies in $K$. It follows that $[s, t]$ and $\left[s, t^{\prime}\right]$ are projective in $K$. Therefore $\Gamma$ so are $\mathfrak{p}$ and $\mathfrak{p}^{\prime}$.

Lemma 7. In $K$, every well-ordered chain with an upper bound is small.

Proof. Let $S$ be a well-ordered chain in $K$ bounded from above by $u \in K$. For each $s \in S$ other than the greatest element of $S$ (if it exists) $\Gamma$ let $s^{+}$be the cover of $s$ in $S \Gamma$ and choose $v_{s} \in s^{+} \backslash s$. It is clear that this produces an independent set of vectors contained in $u$. Since $\operatorname{dim} u<\mathfrak{m} \Gamma$ by (K1) $\Gamma$ it follows that $|S|<\mathfrak{m}$.

\section{The proof of the Theorem}

Having constructed $K$ Twe need to study its lattice of $\mathfrak{m}$-complete congruences. As before $\Gamma c$ ranges over the set $C$ of colors (nonzero m-compact elements of $K$ ). We also let $x$ range over the elements of $L$.

For each $x \in L$ Tdefine an equivalence relation $\Phi^{x}$ on $\mathrm{PG}(W)$ by

$$
s \equiv t \quad\left(\Phi^{x}\right) \text { iff } s^{c}=t^{c} \text { for all } c \not x .
$$

Let $\Theta^{x}$ be the restriction of the relation $\Phi^{x}$ to $K$. We shall prove $\Gamma$ in several steps $\Gamma$ that $L$ is isomorphic to $\operatorname{Con}_{\mathfrak{m}} K$; and this is established by the isomorphism $x \mapsto \Theta^{x}$.

Lemma 8. For $x \in L, \Theta^{x}$ is an $\mathfrak{m}$-complete congruence of $K$.

Proof. Property (4) and Lemma 2 imply that $\Theta^{x}$ is a congruence on $K$. To prove that $\Theta^{x}$ is $\mathfrak{m}$-complete $\Gamma$ assume that $s_{j} \equiv t_{j}\left(\Theta^{x}\right) \Gamma$ for all $j$ in some small nonempty set $J$. Since the meet in $K$ is set intersection $\Gamma$ which is trivial to handle $\Gamma$ we discuss 
only the join. Let $s^{\prime}$ (resp. $s$ ) be the join of $\left(s_{j} \mid j \in J\right)$ in PG(W) (resp. in $K$ ). Define $t^{\prime}$ and $t$ Tsimilarly. Then $s^{\prime} \equiv t^{\prime}\left(\Phi^{x}\right)$ rby (4) and (9).

To prove that $s \equiv t\left(\Theta^{x}\right) \Gamma$ we must consider the process by which $s$ and $t$ are obtained from $s^{\prime}$ and $t^{\prime}$ Trespectively. Define $\Gamma_{s^{\prime}}$ and $\Gamma_{t^{\prime}}$ as $\Gamma_{u^{\prime}}$ was defined in (5). Elements of these sets are ordinals less than $2 \chi$. Also recall from (6) in the proof of Lemma 2 that

$$
s=\left[s^{\prime} \cup \bigcup\left(B_{\gamma} \mid \gamma \in \Gamma_{s^{\prime}}\right)\right]
$$

and similarly for $t$.

If $y \Gamma z \in \operatorname{PG}(W)$ satisfy (K2) and $y \equiv z\left(\Phi^{x}\right) \Gamma$ then for any $\Gamma \subseteq 2 \chi$ we have:

$$
y \vee \bigvee\left(\left[B_{\gamma}\right] \mid \gamma \in \Gamma\right) \equiv z \vee \bigvee\left(\left[B_{\gamma}\right] \mid \gamma \in \Gamma\right) \quad\left(\Phi^{x}\right)
$$

A similar relation holds ifTin addition $\Gamma$ we join another family $\left(\left[B_{\gamma}\right] \mid \gamma \in \Gamma_{1}\right)$ to one side but not to the otherTprovided every $\gamma \in \Gamma_{1}$ satisfies:

$$
B_{\gamma} \subseteq \bigcup\left(B^{c} \mid c \leq x\right) .
$$

So to show that $s^{\prime} \equiv t^{\prime}\left(\Phi^{x}\right)$ Tit suffices to verify that $\gamma$ satisfies condition (12) whenever $\gamma$ lies in exactly one of the sets $\Gamma_{s^{\prime}}$ and $\Gamma_{t^{\prime}}$. If one of $\gamma$ and $\tilde{\gamma}$ satisfies (12) Tthen so does the otherTbecause of the way in which the chains were colored. Indeed $\Gamma$ if $\gamma<\chi$ and $A$ is the set of colors of elements of $B_{\gamma}$ Tthen the elements of $B_{\tilde{\gamma}}$ have color $\bigvee A$. Therefore $\Gamma$ we need only verify (12) for either $\gamma$ or $\tilde{\gamma}$.

Let us suppose that $\gamma \in \Gamma_{t^{\prime}} \backslash \Gamma_{s^{\prime}}$. Then $B_{\gamma} \nsubseteq t^{\prime}$ but $B_{\tilde{\gamma}} \subseteq t^{\prime}$. Also Teither $B_{\gamma}$ and $B_{\tilde{\gamma}}$ are contained in $s^{\prime}$ Tor else neither of them is contained in $s^{\prime}$. Therefore $\Gamma$ possibly after interchanging the roles of $s^{\prime} \Gamma t^{\prime}$ and/or $\gamma \Gamma \tilde{\gamma} \Gamma$ we may assume that $B_{\gamma} \subseteq s^{\prime}$ but $B_{\gamma} \nsubseteq t^{\prime}$. Choose $b \in B_{\gamma}$ with $b \in s^{\prime} \backslash t^{\prime}$.

Now suppose that there is an element $b^{\prime}$ of $B_{\gamma}$ of color $c^{\prime} \nless x$. By (2) $\Gamma$ the definition of the coloring $\Gamma$ we can choose such a $b^{\prime}$ with $b \ll b^{\prime}$. Since $s^{\prime} \equiv t^{\prime}\left(\Phi^{x}\right) \Gamma$ we have $s^{\prime} \cap\left[B^{c^{\prime}}\right]=t^{\prime} \cap\left[B^{c^{\prime}}\right]$; therefore $b^{\prime} \in t^{\prime} \Gamma$ as $b^{\prime} \in s^{\prime} \cap B^{c^{\prime}}$. It follows that $b^{\prime}$ is a linear combination of vectors lying in subspaces of the form $t_{j} \Gamma j \in J$. By (3) $\Gamma$ there exists a $j \in J$ and a vector $v \in t_{j}$ such that $b^{\prime} \in \operatorname{supp} v$. But now $\Gamma(\mathrm{K} 3)$ applied to $t_{j}$ shows that $b$ lies in $t_{j}$ Tand hence also in $t^{\prime}$. This contradicts $b \notin t^{\prime}$. Thus no element $b^{\prime}$ of $B_{\gamma}$ has color $c^{\prime} \nless \leq x$.

This verifies the condition (12) $\Gamma$ as claimed. It follows that the congruence $\Theta^{x}$ of $K$ is $\mathfrak{m}$-complete.

For $\Theta \in \operatorname{Con}_{\mathfrak{m}} K$ Tlet $\mathrm{I}(\Theta)$ denote the set of all colors of prime intervals $[s, t]$ for which $s \equiv t(\Theta)$. By Lemma $6 \Gamma \Theta$ collapses all prime intervals whose color is in $\mathrm{I}(\Theta)$.

Lemma 9. Let $\Theta \in \operatorname{Con}_{\mathfrak{m}} L$, and let $A$ be a small nonempty subset of $C$. Then $\bigvee A \in \mathrm{I}(\Theta)$ iff $A \subseteq \mathrm{I}(\Theta)$.

Proof. For $\gamma<2 \chi \Gamma$ let $C_{\gamma}=\left\{s_{b} \mid b \in B_{\gamma}\right\}$ Гwhere $s_{b}$ was defined in (8). Evidently $C_{\gamma}$ Twith the ordering inherited from $K \Gamma$ is isomorphic to $B_{\gamma} \Gamma$ and it is therefore a small well-ordered chain in $K$. Its prime intervals are of the form $\left[s_{b}, t_{b}\right] \Gamma b \in B_{\gamma}$.

Now choose the $\gamma<\chi$ for which $A_{\gamma}=A$. Let $i_{\gamma}=\left[B_{\gamma} \cup B_{\chi+\gamma}\right]$. It is routine to prove that $i_{\gamma} \in K$ and $\Gamma$ in view of (K4) $\Gamma$

$$
i_{\gamma}=\bigvee C_{\gamma}=\bigvee C_{\chi+\gamma} .
$$


A proof by transfinite induction shows that if $s_{b} \equiv t_{b}(\Theta)$ Tfor all $b \in B_{\gamma} \Gamma$ then $0_{K} \equiv i_{\gamma}(\Theta)$. The converse of this result is trivial. A similar equivalence holds for $B_{\chi}+\gamma$.

The set of colors of the prime intervals of the chain $C_{\gamma}$ is $A$ Twhereas the prime intervals of $C_{\chi+\gamma}$ all have the same color Tnamely $\Gamma \vee A$. By considering the way in which the prime intervals $\left[s_{b}, t_{b}\right]$ were colored $\Gamma$ we can now see that $\bigvee A \in \mathrm{I}(\Theta)$ iff $0_{K} \equiv i_{\gamma}(\Theta)$ Twhich in turn is equivalent to $A \subseteq \mathrm{I}(\Theta)$.

Recall that $\operatorname{Id}_{\mathfrak{m}} C$ is the lattice of all $\mathfrak{m}$-complete ideals of $C$ Ttogether with $\varnothing$.

Lemma 10. The map $\operatorname{Con}_{\mathfrak{m}} K \rightarrow \mathrm{Id}_{\mathfrak{m}} C$ defined by $\Theta \mapsto \mathrm{I}(\Theta)$ is an isomorphism.

Proof. By Lemma 9 and the definition of $\mathfrak{m}$-complete ideals of $C \Gamma \Theta \mapsto \mathrm{I}(\Theta)$ maps $\operatorname{Con}_{\mathfrak{m}} K$ into $\mathrm{Id}_{\mathfrak{m}} C$. Moreover $\Gamma$ this map is order-preserving. Now let us assume that $\Theta$ and $\Psi$ are distinct $m$-complete congruences of $K$ Tsay $\Theta \nsubseteq \Psi$. Choose an interval $[s, t]$ of $K$ collapsed by $\Theta$ but not by $\Psi$. By Lemma 7 and Zorn's LemmaT there is in $K$ a maximal well-ordered chain from $s$ to $t \Gamma$ of order type less than $\mathfrak{m}$. In this chainT let $u$ be the least element which satisfies $s \not \equiv u(\Psi)$. Since $\Psi$ is

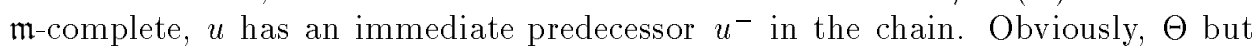
not $\Psi$ collapses the prime interval $\left[u^{-}, u\right]$. Thus $\mathrm{I}(\Theta) \nsubseteq \mathrm{I}(\Psi)$. It also follows that the map is injective.

Next observe that $\Gamma$ for $x \in L \Gamma \mathrm{L}\left(\Theta^{x}\right)=\{c \in C \mid c \leq x\}$ Tas can be seen from the definition of $\Theta^{x}$ and the fact that every $c \in C$ is the color of some prime interval. Thus by Lemma 1 the map is onto.

The Theorem is an immediate consequence of the previous lemma and Lemma 1. The isomorphism $L \cong \operatorname{Con}_{\mathfrak{m}} K$ is given by $x \mapsto \Theta^{x}$.

\section{Concluding comments}

This lattice $K$ we constructed for the Theorem has many special properties.

(i) The lattice $K+1$ ( $K$ with a unit element adjoined) is a complete lattice.

(ii) $K$ is not only modularTit is a sublattice of the lattice of subspaces of a vector space; therefore it is arguesian.

(iii) $K$ has a type 1 representation (see $\Gamma$ for instance $\Gamma[3] \Gamma$ p. 198).

(iv) $K$ has a zero element 0 Tand there is a one-to-one correspondence $\Theta \mapsto[0] \Theta$ between $\mathfrak{m}$-complete congruences of $K$ and $\mathfrak{m}$-complete congruence kernel ideals.

In [1] TR. Freese TW. A. LampeTand W. Taylor proved an interesting result on the representation of algebraic lattices as congruence lattices: For every cardinal $\mathfrak{n}$, there is an algebraic lattice $L$ which cannot be represented as the congruence lattice of a finitary algebra with fewer than $\mathfrak{n}$ operations.

We would like to point out that the case of infinitary algebras is different.

Theorem . Let $\mathfrak{m}$ be a cardinal of the form $\aleph_{\alpha+1}$. Every $\mathfrak{m}$-algebraic lattice $L$ is isomorphic to the congruence lattice of an algebra with a single operation, of arity $\aleph_{\alpha}$.

Proof. We can regard the lattice $K$ constructed in Section 3 as an algebra

$$
\left\langle K ;\left\{\bigvee_{\mathfrak{m}}, \bigwedge_{\mathfrak{m}}\right\}\right\rangle
$$

where the arity of the operations $\bigvee_{\mathfrak{m}}$ and $\bigwedge_{\mathfrak{m}}$ is $\aleph_{\alpha}$. This algebra is polynomially equivalent to an algebra $\langle K ; f\rangle \Gamma$ where the operation $f \Gamma$ of arity $\aleph_{\alpha} \Gamma$ is defined below. 
We can write $I=\left\{\gamma \mid \gamma<\aleph_{\alpha}\right\}$ as a disjoint union $I=\bigcup \bigcup U\left(I_{i} \mid i<\aleph_{\alpha}\right) \Gamma$ where each $I_{i}$ is a set of cardinality $\aleph_{\alpha}$. Now define

$$
f(\mathbf{x})=\bigvee_{\mathfrak{m}}\left(\bigwedge_{\mathfrak{m}}\left(\mathbf{x}_{\mathbf{j}} \mid \mathbf{j} \in \mathbf{I}_{\mathbf{i}}\right) \mid \mathbf{i}<\aleph_{\alpha}\right),
$$

where $\mathbf{x}=\left\langle\mathbf{x}_{\mathbf{0}}, \mathbf{x}_{1}, \ldots, \mathbf{x}_{\gamma}, \ldots\right\rangle \Gamma \gamma<\aleph_{\alpha}$.

Obviously any cardinal $\mathfrak{n}<\aleph_{\alpha} \Gamma$ there is an $\mathfrak{m}$-algebraic lattice $L$ that cannot be represented as the congruence lattice of an algebra $\langle A ; F\rangle$ where each $f \in F$ has arity $\leq \mathfrak{n}$.

For example Ttake a set $J$ that is not small and let $L$ be the lattice of all subsets of $J$ whose complement is small $\Gamma$ together with the empty set.

\section{REFERENCES}

[1] R. Freese, W. A. Lampe, and W. Taylor, Congruence lattices of algebras of fixed similarity types. I., Indag. Math. 82 (1) (1979), 59-68.

[2] G. Grätzer, On a family of certain subalgebras of a universal algebra, Indag. Math. 68 (1965), $790-802$.

[3] G. Grätzer, General Lattice Theory, Academic Press, New York, N. Y., Birkhäuser Verlag, Basel, Akademie Verlag, Berlin, 1978.

[4] G. Grätzer, Universal Algebra, Springer-Verlag, New York-Heidelberg-Berlin, second ed.,1979.

[5] G. Grätzer, The complete congruence lattice of a complete lattice, Lattices, Semigroups, and Universal Algebra, Proceedings of an international conference on lattices, semigroups, and universal algebra (Lisbon, 1988), Plenum Press, New York and London, 1990, pp. 81-88.

[6] G. Grätzer and E. T. Schmidt, Algebraic lattices as congruence lattices: The $\mathfrak{m}$-complete case, Birkhoff Conference (Darmstadt, 1991), to appear.

[7] K. Reuter and R. Wille, Complete congruence relations of complete lattices, Acta Sci. Math. (Szeged) 51 (1987), 319-327.

[8] S.-K. Teo, Representing finite lattices as complete congruence lattices of complete lattices, Ann. Univ. Sci. Budapest. Eötvös Sect. Math. 33 (1990),177-182.

UNIVERSITY OF MANITOBA

Department of Mathematics

Winnip EG, Man. R3T 2N2

CANADA

E-mail address, G. Grätzer: gratzer@ccm.umanitoba.ca

P. M. Johnson: pmjeccu.umanitoba.ca

TeChnical University of Budafest

Transport EngineERIng FaCUlty

Defartment of Mathematics

1111 BUDAPEST

MÜEGYeTEM RKP. 9

HUNGARY

E-mail address, E. T. Schmidt: h1175sch@ella.hu 